\title{
Trombectomía mecánica, aspirativa e intra- arterial. Primer caso en un paciente con stroke isquémico agudo de un hospital nacional
}

\author{
Intra-arterial, aspirate and mechanical thrombectomy. First case in a patient with an acute stroke in a \\ hospital
}

\author{
Giancarlo Saal-Zapata ${ }^{1, a}$, Rodolfo Rodríguez ${ }^{1, a}$ \\ RESUMEN
}

El stroke isquémico agudo es una de las principales causas de morbilidad y mortalidad en el mundo. Si existe compromiso de vaso grande, la trombectomía mecánica es la técnica endovascular que ha demostrado los mejores resultados. Presentamos el caso de un paciente con trombosis de la arteria cerebral media izquierda de 5 horas de evolución, con afasia mixta y hemiplejía, la escala ASPECTS 7, score NIHSS 14 y escala de Rankin modificada (mRS) de 4. Se realizó trombectomía mecánica con stents Solitaire AB y ERIC, aspiración manual de coágulo con jeringa de $60 \mathrm{cc}$ e instilación de alteplase intra-arterial. La reperfusión inmediata fue total (TICI 3). El mRS posterior al tratamiento fue de 2 .

PALABRAS CLAVE: Infarto cerebral, trombectomía, stent retriever. (Fuente: DeCS BIREME).

\section{SUMMARY}

Acute stroke is one of the most common causes of morbidity and mortality worldwide. Mechanical thrombectomy is the endovascular procedure that has shown better results in patients with a large vessel involvement. We present here a case of a patient who had a five-hour history of thrombosis of the left mid cerebral artery who developed mixed aphasia and hemiparesis, ASPECTS scale of five, NIHSS score of 14 and a modified Rankin scale (mRS) of 4. A mechanical thrombectomy was performed with stents Solitaire AB and ERIC and manual aspiration of the clot with a $60 \mathrm{cc}$ syringe plus instillation of intra-arterial alteplase. Immediate reperfusion was complete (TICI 3). The $\mathrm{mRS}$ after the procedure was 2 .

KEYWORDS: Cerebral infarction, thrombectomy, stent retriever. (Source: MeSH NLM).

\section{INTRODUCCIÓN}

El stroke isquémico agudo representa el $85 \%$ del total de los ictus y es la segunda causa de muerte y tercera de discapacidad en el mundo $(1,2)$. En el Perú, se ha reportado una mortalidad de 19,6\% (3).

Una vez realizado el diagnóstico, basado en el cuadro clínico y las imágenes en la tomografía o en la resonancia cerebral, se debe realizar el tratamiento el cual dependerá del tiempo transcurrido desde el inicio del evento (4). La trombolisis endovenosa está indicada antes de las 4,5 horas. Si existe oclusión de vaso grande (arteria carótida interna, cerebral media, cerebral anterior), se recomienda la trombectomía mecánica, que ha demostrado altas tasas de reperfusión y mejores resultados funcionales evaluados con la escala de Rankin modificada (mRS) a los 90 días

1 Departamento de Neurocirugía, Servicio de Neurocirugía Endovascular. Hospital Nacional Guillermo Almenara Irigoyen,

EsSalud. Lima, Perú.

a Neurocirujano. 
comparado con el manejo médico (5-9). Actualmente, el periodo de ventana de la trombectomía mecánica se considera hasta las 24 horas, siguiendo estrictos criterios de inclusión $(10,11)$.

En nuestro país no se conoce la proporción de pacientes con stroke isquémico agudo de vaso grande, que son sometidos a trombectomía mecánica, esto se suma a que no todos los centros hospitalarios cuentan con un equipo de médicos entrenados y la infraestructura necesaria para realizar este tipo de procedimiento.

Por ello, se reporta el primer caso de stroke isquémico agudo de vaso grande tratado con trombectomía en sus tres modalidades: mecánica, aspirativa e intra-arterial.

\section{PRESENTACIÓN DEL CASO}

Varón de 70 años con antecedente de hipertensión arterial, infarto de miocardio y fibrilación auricular en tratamiento con losartán, aspirina y cilostazol.

Ingresó a nuestra institución referido de un hospital de la capital con un tiempo de enfermedad de 5 horas, caracterizado por afasia y hemiplejia derecha, siendo catalogado con un score NIHSS de 14 puntos y escala de Rankin modificada (mRS) de 4 puntos.

Se le realizó TEM cerebral donde se evidenció "signo de la cuerda" en la arteria cerebral media izquierda y escala ASPECTS de 7 puntos (figura 1). Bajo anestesia general, se realizó punción femoral derecha con introductor 9F, se navegó con catéter
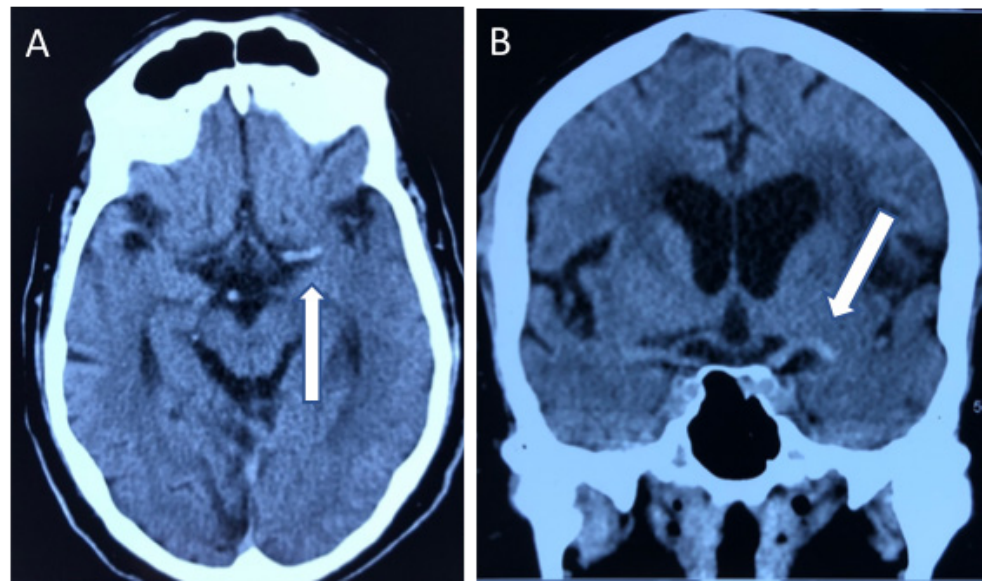

Figura 1. Tomografía espiral multicorte sin contraste, demostrando "signo de la cuerda" en arteria cerebral media izquierda (flecha blanca) en corte axial (A) y coronal (B).
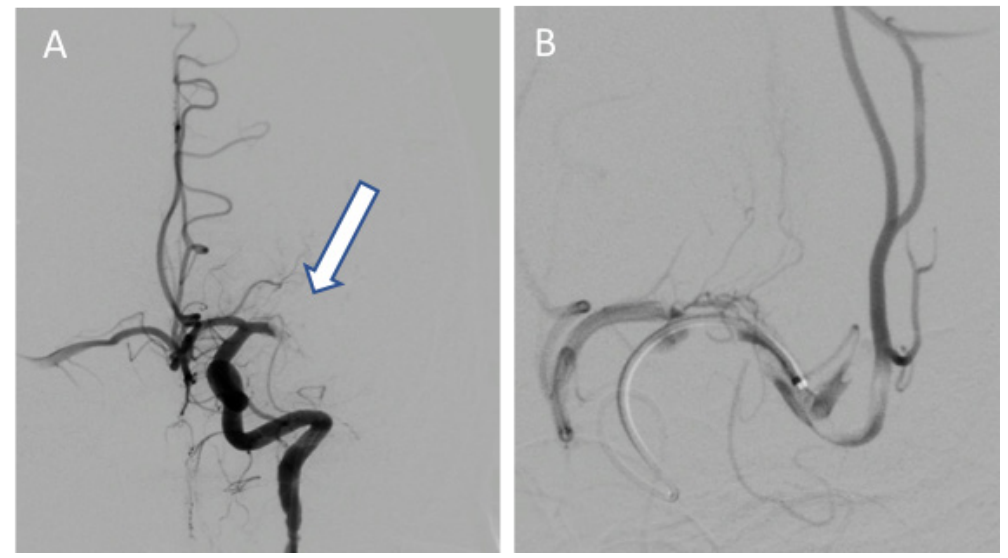

Figura 2. A: Angiografía por sustracción digital demostrando ausencia de arteria cerebral media izquierda (flecha blanca) a partir del segmento M1 en vista frontal. B: Angiografía ultra selectiva de arteria cerebral media izquierda en segmento M2 evidenciando presencia de trombos. 
guía $5 \mathrm{~F}$ y se realizó angiografía de arteria carótida común izquierda, confirmando la ausencia de arteria cerebral media (ACM) ipsilateral, luego se realizó una angiografía ultra selectiva evidenciándose un trombo en el segmento M1 (figura 2). Se realizó intercambio de catéteres y se navegó con catéter guía de $8 \mathrm{~F}$ hasta la arteria carótida interna (ACI) izquierda seguido de un catéter de acceso distal. Se navegó con microcatéter de 0,028 pulgadas y microguía de 0,014 pulgadas hasta el segmento M2 de la ACM izquierda, luego se utilizó stent retractor en dos ocasiones (figura 3), no obteniendo retracción del coágulo. Se decidió utilizar el stent ERIC (Embolus Retriever with Interlinked Cages) con microcatéter de 0,017 pulgadas: con la primera pasada se extrae coágulo (figura 4 A y B), se decidió realizar una segunda pasada siendo exitosa. Posteriormente se aspiró manualmente con jeringa de $60 \mathrm{cc}$ y microcatéter de 0,029 pulgadas (figura 4C), obteniendo fragmentos de coágulo. Por último, se administraron $5 \mathrm{cc}$ de alteplasa a nivel de M1 izquierda como terapia adjunta a la trombectomía. En el control final se observó reperfusión total de la arteria comprometida (TICI 3) (figura 5). Fue dado de alta con un mRS de 2.
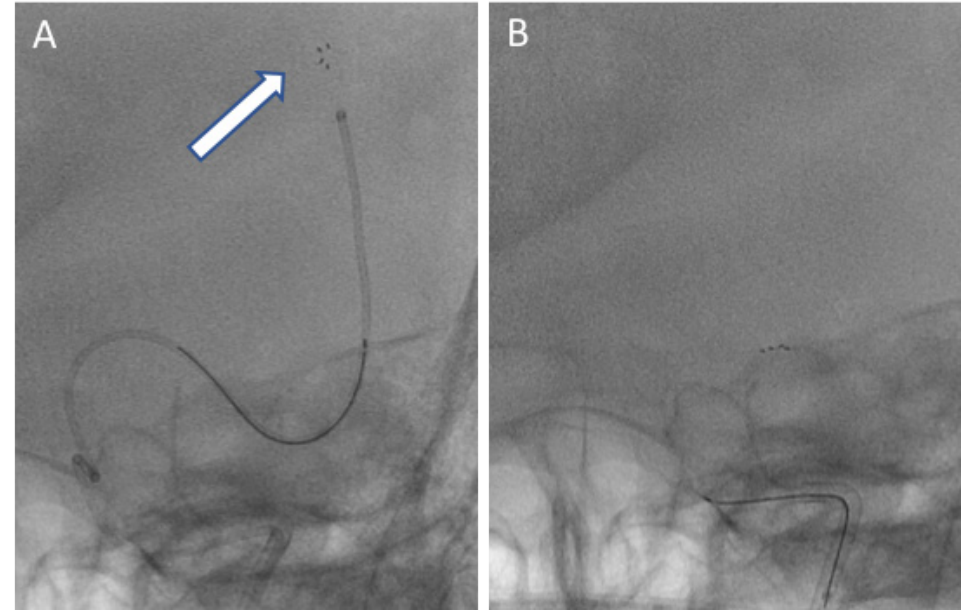

Figura 3. (A) Microcatéter de 0,029 pulgadas navegando en segmento de arteria M2 izquierda y stent retractor siendo desplegado (flecha blanca). (B) Retracción del stent a nivel del segmento M1 izquierdo.
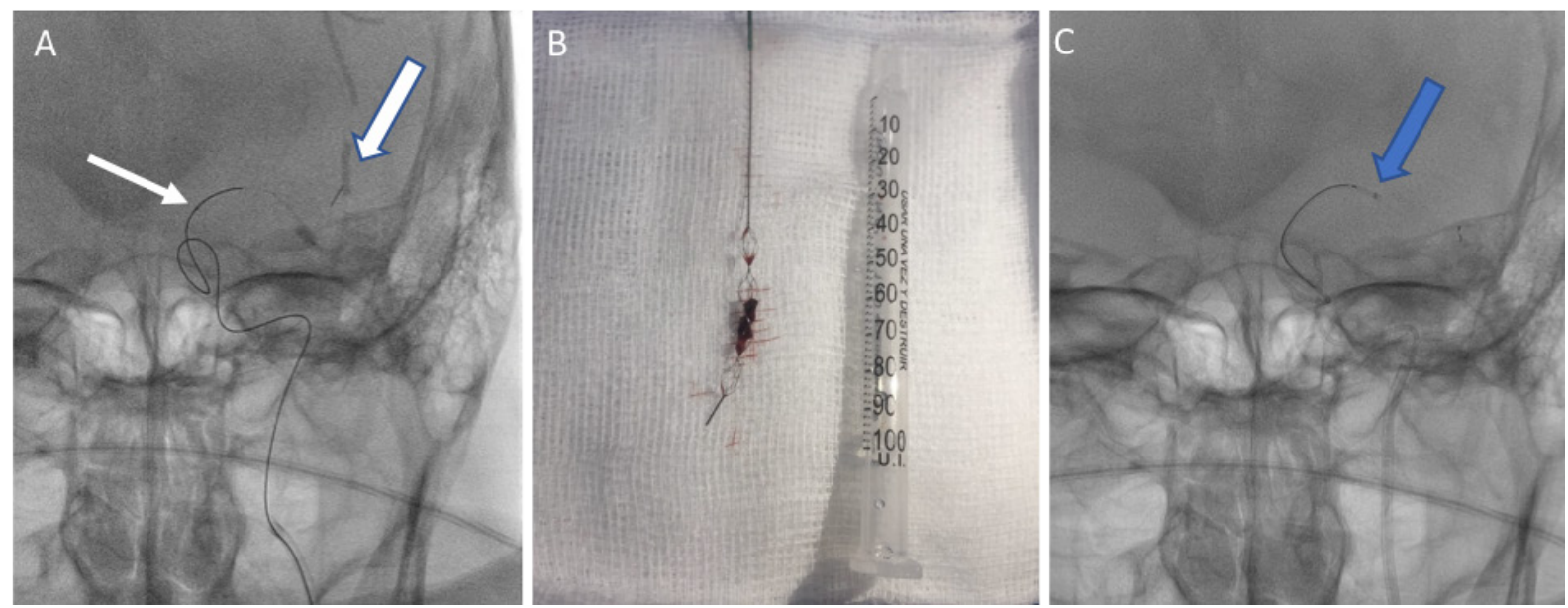

Figura 4. A: Microcatéter de 0,017 pulgadas (flecha blanca delgada) navegando en segmento M2 izquierdo: se observa stent ERIC siendo desplegado (flecha blanca gruesa). Nótese la marca distal que es diferente al stent retractor de primera generación. B: Stent ERIC con coágulo retraído. C: Microcatéter de 0,029 pulgadas en segmento M1 aspirando coágulo (flecha azul). 


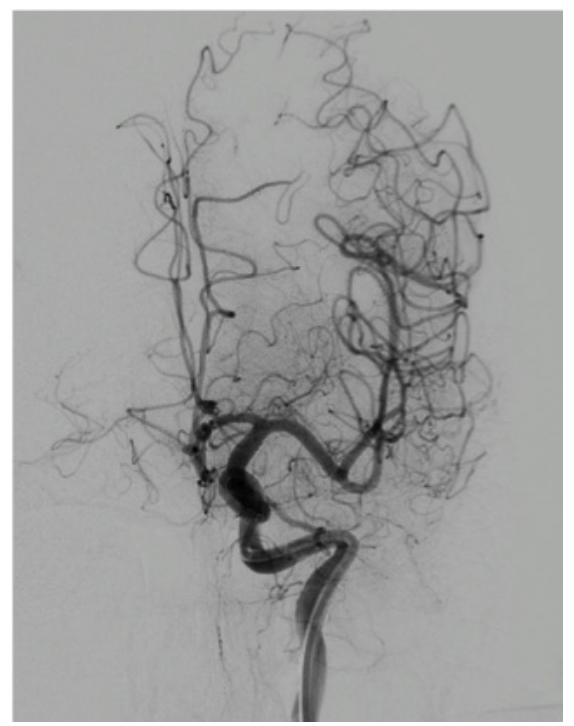

Figura 5. Reperfusión total de arteria cerebral media izquierda (TICI 3): se observan las 2 ramas de la bifurcación de la ACM izquierda y ramas distales previamente ocluidas.

\section{DISCUSIÓN}

El manejo actual del stroke isquémico agudo por oclusión de vaso grande es endovascular, siendo la trombectomía mecánica la técnica de elección (12).

La trombolisis endovenosa era hasta hace unos años la única alternativa en estos pacientes y está indicada si el paciente tiene un tiempo de evolución del cuadro clínico de 4,5 horas (13). Su efecto trombolítico en oclusión de vaso grande es pobre (14).

En el año 2015, cinco estudios randomizados demostraron altas tasas de reperfusión y mejores resultados funcionales cuando se realizaba la trombectomía mecánica en el stroke isquémico agudo de vaso grande comparado con el tratamiento médico utilizando el alteplasa, fármaco activador tisular del plasminógeno recombinante, administrado por vía endovenosa, con mejoría en la independencia funcional a los 90 días evaluado por la mRS (5-9). Todos los procedimientos se realizaron antes de las 6 horas de iniciado el evento y utilizaron tomografía o resonancia para evaluar la extensión del infarto. La ventana de tiempo para realizar la trombectomía clásicamente era de 6 horas, sin embargo, actualmente este periodo se ha extendido hasta las 24 horas.

Dos estudios han demostrado buenos resultados funcionales cuando la trombectomía mecánica se realiza hasta las 16 horas (10) y hasta las 24 horas (11), dependiendo del cumplimiento de estrictos criterios de imágenes, empleando secuencias de difusión y perfusión en tomografía y resonancia magnética cerebral.

Existen diferentes stents recuperadores de coágulo: los de primera generación $(16,17)$ y posteriormente se creó el stent ERIC (Embolus Retriever with Interlinked Cages) de segunda generación (18). Estos dispositivos endovasculares tienen la capacidad de compactar el coágulo en sus celdas al desplegarse dentro del vaso y luego de unos minutos ser retraído.

En la TEM cerebral del paciente reportado, se evidenciaba el signo de la cuerda, también llamado signo de la arteria cerebral media hiperdensa, que orienta a un trombo cuya composición es predominante de glóbulos rojos (15). La escala ASPECTS fue de 7 y el score NIHSS fue de 14. Era evidente una oclusión de vaso grande y el paciente ingresó directamente a la sala de angiografía para realizar la trombectomía mecánica.

En nuestro caso, no se empleó trombolisis endovenosa porque el tiempo transcurrido desde el inicio del evento hasta la llegada a nuestra institución fue mayor de 4,5 horas. Sin embargo, se utilizó un stent retractor de coágulo de primera generación, el cual navega dentro de un microcatéter con un diámetro interno mínimo de 0,021 pulgadas. Al ser frustra la extracción del trombo, utilizamos el stent ERIC, que tiene las ventajas de ser navegado dentro de un microcatéter de 0,017 pulgadas y tener una morfología que permite generar menor fragmentación y menor contacto con la pared del vaso (18). Posteriormente, se realizó trombectomía aspirativa manual con jeringa de $60 \mathrm{cc}$, la cual ha demostrado resultados satisfactorios de repermeabilización (19). Finalmente, se realizó trombolisis intra-arterial con alteplase como terapia adjunta a la trombectomía, habiendo reportes de su empleo con éxito (20).

Este es el primer caso en el que se realiza trombectomía mecánica, aspirativa e intra-arterial en un paciente con stroke isquémico agudo de vaso grande en nuestra institución.

Una limitación de este reporte fue no poder evaluar clínicamente y hacer seguimiento del paciente después del alta por problemas administrativos. Sin embargo, al alta presentó un mRS de 2 . 


\section{Declaración de financiamiento y conflictos de interés:}

Reporte fue financiado por los autores. No existe conflicto de intereses de parte de los autores ni de orden personal, económico, institucional o laboral.

\section{Contribución de autoría:}

GSZ: Concepción y redacción del artículo, recolección e interpretación de los datos, cirujano participante del procedimiento y aprobación de la versión final. RR: Cirujano participante del procedimiento y aprobación de la versión final.

\section{Correspondencia:}

Giancarlo Saal-Zapata

Avenida Grau 800, La Victoria, Lima, Perú.

e-mail: gian_carlo1987@hotmail.com

\section{REFERENCIAS BIBLIOGRÁFICAS}

1. Davalos LF, Málaga G. El accidente cerebrovascular en el Perú: una enfermedad prevalente olvidada y desatendida. Rev Peru Med Exp Salud Publica. 2014; $31: 400-1$.

2. O’Donnell MJ, Chin SL, Rangarajan S, et al. Global and regional effects of potentially modifiable risk factors associated with acute stroke in 32 countries (INTERSTROKE): a case-control study. Lancet. 2016; 388(10046):761-75.

3. Castañeda-Guarderas A, Beltrán-Ale G, CasmaBustamante R, Ruíz-Grosso P, Málaga G. Registro de pacientes con accidente cerebro vascular en un hospital público del Perú, 2000-2009. Rev Peru Med Exp y Salud Pública; 2011; 28(4):623-7.

4. Yoo AJ, Andersson T. Thrombectomy in acute ischemic stroke: Challenges to procedural success. J Stroke. 2017; 19(2):121-30.

5. Jovin TG, Chamorro A, Cobo E, et al. Thrombectomy within 8 Hours after Symptom Onset in Ischemic Stroke. N Engl J Med. 2015; 372(24):2296-306.

6. Saver JL, Goyal M, Bonafe A, et al. Stent-Retriever Thrombectomy after Intravenous t-PA vs. t-PA Alone in Stroke. N Engl J Med. 2015; 372(24):2285-95.

7. Goyal M, Demchuk AM, Menon BK, et al. Randomized Assessment of Rapid Endovascular Treatment of Ischemic Stroke. N Engl J Med. 2015; 372(11):1019-30.

8. Campbell BC V, Mitchell PJ, Kleinig TJ, et al. Endovascular Therapy for Ischemic Stroke with Perfusion-Imaging Selection. N Engl J Med. 2015; 372(11):1009-18
9. Berkhemer OA, Fransen PSS, Beumer D, et al. A Randomized Trial of Intraarterial Treatment for Acute Ischemic Stroke. N Engl J Med. 2014; 372(1):11-20.

10. Albers GW, Marks MP, Kemp S, et al. Thrombectomy for Stroke at 6 to 16 Hours with Selection by Perfusion Imaging. N Engl J Med. 2018; 378(8):708-18.

11. Nogueira RG, Jadhav AP, Haussen DC, et al. Thrombectomy 6 to 24 Hours after Stroke with a Mismatch between Deficit and Infarct. N Engl J Med. 2017; 378(1):11-21.

12. Jansen IGH, Mulder MJHL, Goldhoorn R-JB. Endovascular treatment for acute ischaemic stroke in routine clinical practice: prospective, observational cohort study (MR CLEAN Registry). BMJ. 2018; 360:k949.

13. Hacke W, Kaste M, Bluhmki E, et al. Thrombolysis with Alteplase 3 to 4.5 Hours after Acute Ischemic Stroke. N Engl J Med. 2008; 359(13):1317-29.

14. Campbell BC V, Mitchell PJ, Churilov L, et al. Tenecteplase versus Alteplase before Thrombectomy for Ischemic Stroke. N Engl J Med. 2018; 378(17):1573-82.

15. Liebeskind DS, Sanossian N, Yong WH, et al. CT and MRI early vessel signs reflect clot composition in acute stroke. Stroke. 2011; 42(5):1237-43. doi: 10.1161/STROKEAHA.110.605576

16. Campbell BC, Hill MD, Rubiera M, et al. Safety and Efficacy of Solitaire Stent Thrombectomy: Individual Patient Data Meta-Analysis of Randomized Trials. Stroke. 2016; 47(3):798-806. doi: 10.1161/ STROKEAHA.115.012360

17. Zaidat OO, Castonguay AC, Nogueira RG, et al. TREVO stent-retriever mechanical thrombectomy for acute ischemic stroke secondary to large vessel occlusion registry. J Neurointerv Surg. 2018; 10(6):516-524.

18. Steglich-Arnholm H, Kondziella D, Wagner A, et al. Mechanical Thrombectomy with the Embolus Retriever with Interlinked Cages in Acute Ischemic Stroke: ERIC, the New Boy in the Class. Am J Neuroradiol. 2017; 38(7):1356-1361.

19. Kim YS, Kwak HS, Chung GH, Hwang SB. Manual aspiration thrombectomy using the Penumbra catheter in patients with acute M1 occlusion: A singlecenter study. Interv Neuroradiol. 2015; 21(6):694-9.

20. Heiferman DM, Li DD, Pecoraro NC, Smolenski AM, Tsimpas A, Ashley Jr WW. Intra-Arterial Alteplase Thrombolysis during Mechanical Thrombectomy for Acute Ischemic Stroke. J Stroke Cerebrovasc Dis. 2017; 26(12):3004-8.

Recibido: $19 / 06 / 2019$

Aceptado: 27/12/2019 